\title{
Development of Fuzzy Level of Service Criteria for Bus Rapid Transit considering User Heterogeneities in China
}

\author{
Yueying Huo $\mathbb{D D}^{1}{ }^{1}$ Jianrong Liu, ${ }^{2}$ Jian Zhang $\mathbb{D}^{3}{ }^{3}$ and Xiaojuan $\mathrm{Li}^{1}$ \\ ${ }^{1}$ Transportation Institute, Inner Mongolia University, 24 Zhaojun Rd, Hohhot, Inner Mongolia 010070, China \\ ${ }^{2}$ School of Civil Engineering and Transportation, South China University of Technology, 381 Wushan Rd, Guangzhou, \\ Guangdong Province 510640, China \\ ${ }^{3}$ School of Transportation, Southeast University, 2 Southeast University Rd, Nanjing, Jiangsu Province 211189, China \\ Correspondence should be addressed to Yueying Huo; hyy@imu.edu.cn
}

Received 26 March 2020; Revised 23 May 2020; Accepted 11 June 2020; Published 14 July 2020

Academic Editor: Yanyong Guo

Copyright () 2020 Yueying Huo et al. This is an open access article distributed under the Creative Commons Attribution License, which permits unrestricted use, distribution, and reproduction in any medium, provided the original work is properly cited.

\begin{abstract}
Level of service (LOS) analysis based on LOS criteria is essential for the planning, design, and operational evaluation of public transit. However, there are no systematic transit LOS criteria at present in China. Bus rapid transit (BRT) is receiving increasing attention worldwide. Therefore, this study addresses LOS criteria for BRT in China. Transit passengers are heterogeneous in their perceptions, needs, and behavior. The traditional hard LOS criteria have an inherent weakness, because of which the accuracy of an LOS analysis is limited. Thus, in this study, we initially conducted transit market segmentation to reduce heterogeneity and subsequently developed BRT fuzzy LOS criteria for different passenger groups. Using a smartphone-based transit travel survey system, we organized BRT passenger travel surveys on three BRT systems in China to collect data. Transit market segmentation was performed based on user perceptions; passengers were segmented into a calm passenger group and an anxious passenger group using the latent class model. Passenger arrival time, passenger wait time, and running speed of the bus were selected as service metrics to reflect the BRT's LOS. BRT fuzzy LOS criteria for the three service metrics in the case of both the calm and anxious passenger groups were developed using fuzzy C-means clustering. The LOS criteria for the two groups of passengers fit their psychological characteristics and reflected their personalized travel needs. Fuzzy LOS criteria can describe to what extent service metric values belong to the adjacent LOS categories via the use of membership. Thus, fuzzy LOS criteria can overcome the weakness of hard LOS criteria.
\end{abstract}

\section{Introduction}

Level of service (LOS) criteria using service metrics that classify them into several categories according to different thresholds can provide quantitative LOS analysis standards for transportation systems [1, 2]. LOS analysis based on LOS criteria is very essential for the planning, design, and operational evaluation of transportation facilities or services. Additionally, it is critical for the allocation of limited financial resources to competing transportation projects [1-3]. Transit capacity and quality of service manuals (TCQSMs) provide LOS criteria for public transit services. However, the LOS criteria in the TCQSMs are more suitable for North America, because that is where most of the research is conducted $[4,5]$. In China, there are no systematic transit LOS criteria at present. Therefore, it is necessary to develop transit LOS criteria specific to China in order to implement the LOS analysis.

The bus rapid transit (BRT) combines the efficiency and reliability of a rail service with the operating flexibility and low cost of a conventional bus service [6]. Currently, 168 cities worldwide have constructed BRT corridors and their total length has reached 4998 kilometers [7]. The topic of BRT LOS is receiving increasing attention, and we are carrying out research on it funded by the National Nature Science Foundation of China. Thus, we address the issue of BRT LOS criteria in China.

Transit passengers are heterogeneous [8]. Passenger behavior, need, and perception vary across different groups of passengers. Transit market segmentation, which defines 
specific subgroups of passengers sharing similar demographic, psychographic, or behavioral characteristics, can reduce passenger heterogeneity $[9,10]$. It permits transit providers to comprehend the special travel needs of certain subgroups so that they can cater to personalized travel requirements [9]. Thus, in this study, we will initially carry out transit market segmentation and subsequently develop BRT LOS criteria for different passenger groups.

LOS criteria in the TCQSMs or highway capacity manuals (HCMs) use clear-cut boundaries to divide multiple LOS categories $[1,2,4,5]$, in which a certain value of a service metric exclusively belongs to one LOS category, known as the hard LOS criteria in this study. An inherent weakness of the hard LOS criteria is that certain small changes in service metrics can cause a change in the LOS, while certain significant changes in service metrics may not lead to a variation in LOS. This limits the accuracy with which transportation facilities or services may be characterized $[11,12]$. In order to overcome this weakness of the hard LOS criteria, we will try to develop fuzzy LOS criteria for the BRT in China.

The intent of this research work is to develop BRT fuzzy LOS criteria for passengers grouped using transit market segmentation in China. The research results can be used to implement transit LOS analysis and further guide the planning, design, and operational evaluation of transit services in China. The research methodology can act as a reference source for other regions in developing their own transit LOS criteria.

This paper is organized as follows. A literature review is first presented. Next, data collection, including the development of a transit travel survey system and BRT passenger travel surveys, is described. After this step, the methodology involved in a latent class model and fuzzy C-means clustering are introduced. Finally, the results from transit market segmentation and BRT fuzzy LOS criteria are analyzed and conclusions are summarized.

\section{Literature Review}

The LOS criteria provided by TCQSMs for bus transit with service metrics of frequency, service span, access, passenger load, on-time performance, headway adherence, and transitauto travel time ratio are divided into multiple levels $[4,5]$. Transit passengers are demographically, psychologically, and behaviorally heterogeneous [8]. However, the TCQSMs did not consider the heterogeneity of passengers and provided a set of general LOS criteria for all classes of passengers. Transit market segmentation can reduce passenger heterogeneity and permit transit providers to comprehend the special travel needs of certain subgroups so that they can cater to personalized travel requirements [9].

The most basic form of transit market segmentation is through social demographic and travel characteristics. Dell'Olio et al. stratified passengers by socioeconomic characteristics such as gender, age, and income. Further, models of service quality for different types of passengers were established using ordered probit models. This study demonstrated that the user perception of service quality changed depending on the types of passengers [13]. Oña et al. addressed heterogeneity in user perceptions of service quality for railway service. Users were divided into different groups according to travel purpose, day of journey, and frequency of use. Based on questionnaire surveys conducted in Milan, northern Italy, models for different groups of users were established. This study again found that perceptions about service quality were different among various groups of users [14]. Using the data collected from questionnaire surveys in Kaohsiung, Cheng and Liu examined users' perceptions of intermodal inconvenience for the bicycletransit service by utilizing the Rasch model. Various subgroups of users were defined based on socioeconomic and travel characteristics. This study demonstrated that users' perceived inconveniences differed according to gender, riding frequency, trip purpose, and environmental awareness [15]. Cheng and Chen evaluated public transportation service chains along the three dimensions of accessibility, mobility, and connectivity from the perspective of urban travelers. The analysis based on the Rasch model indicated that young passengers, environmentally conscious users, passengers using public transportation as their main transportation mode, and those who indulged in frequent sporting activities were better able to overcome difficulties during their trips [16]. Bordagaray et al. used ordered probit models to model bus transit quality in the city of Santander considering user and service heterogeneity. The perceived quality models were developed by introducing systematic variations in the users' perceptions to account for various factors such as age, income, and frequency of service use. The results show that heterogeneity is clearly present in the perception of service quality [17]. Passenger segmentation by social demographic and travel characteristics defines classes of passengers but not market segments. It gives rise to unbalanced segments where passengers do not share similar interests, needs, or locations, i.e., heterogeneity among users is still evident [9].

With the proliferation of automated data collection systems such as smart card automated fare collection (AFC), transit market segmentation can be performed with AFC data. Lathia et al. identified behavioral differences between passengers using AFC data. They applied agglomerative hierarchical clustering to segment passengers using temporal travel patterns. A number of algorithms based on historical travel time data were proposed for personalized trip time estimations for different types of passengers. By applying these algorithms to the data from the oyster fare collection system, London, it was empirically demonstrated that these algorithms outperformed both a nonpersonalized baseline computed from the data, as well as published travel times as currently offered by the transport authority [18]. There is a need for an integrated market segmentation method that incorporates both the spatial and behavioral features of individual transit passengers. Kieu et al. proposed a new algorithm named spatial affinity propagation (SAP) based on the classical affinity propagation algorithm (AP) to enable transit market segmentation with spatial-behavioral features. SAP was applied to a 40-day AFC dataset from New South Wales, Australia. This case study on a limited sample 
size demonstrated that AP and SAP were better candidates for spatial passenger segmentation than $K$-means and complete-linkage hierarchical agglomerative clustering. Further, SAP was 52\% more efficient in computation time than AP. The case study carried out on the entire dataset showed that SAP could deal with large-scale AFC data for transit market segmentation [9].

Some studies use the latent class model to conduct transit market segmentation using social demographic, travel, and behavioral characteristics as manifest variables. Oña et al. employed the latent class model to study how bus passengers could be stratified. Based on the data from customer satisfaction surveys in Granada, four groups of passengers were identified by the manifest variables of age, gender, travel reason, use frequency, possession of private vehicle, and ticket. Next, a Pearson correlation was applied for each group to derive the importance of the service quality attributes. It was discovered that the most important attributes for each group were different [10]. Qiao et al. addressed the issue of passenger market segmentation for high-speed railway service in China. The latent class model was used for passenger segmentation using age, gender, travel date, travel distance, mean time to get a ticket, and prepurchase time as manifest variables. Based on the ticket data of the BeijingShanghai high-speed railway, three distinct groups were identified via segmentation. They were the leisure passengers, planned passengers, and temporary passengers [19], respectively. Bellizzi et al. used the latent class model to examine the heterogeneity in the desired bus service quality from the current users' and potential users' perspective. Based on the preference data collected from stated preference surveys, current users were divided into three latent classes. These three classes accorded more importance to journey time (30\% of users), comfort ( $60 \%$ of users), and fare ( $10 \%$ of users), respectively. However, potential users were segregated into two latent classes, and they conferred more importance to journey time ( $47 \%$ of users) and fare (53\% of users) [20], respectively.

The TCQSMs provided hard LOS criteria for bus transit by the use of clear-cut boundaries in the division of various LOS categories $[4,5]$. The HCMs provided hard LOS criteria for various transportation facilities from the intersection to the freeway $[1,2]$. An inherent weakness of the hard LOS criteria is that some small changes in service metrics can cause a change in the LOS. On the other hand, even certain significant changes in service metrics may not lead to a change in LOS, which limits the accuracy with which transportation facilities or services may be characterized $[11,12]$. By contrast, Fang et al. and Fang and Pecheux addressed fuzzy LOS criteria for signalized intersections. They developed a methodology using fuzzy C-means clustering to define fuzzy LOS criteria based on user perception. Using a user perception database of 100 subjects assessing 24 approaches in terms of estimated delay and rating of LOS, the fuzzy LOS criteria for signalized intersections were proposed. Compared with the hard LOS criteria of signalized intersections in the HCMs, fuzzy LOS criteria are capable of expressing the delays near the boundaries of each LOS category $[11,12]$.
In summary, because of the heterogeneity in transit passengers, we should perform transit market segmentation when studying transit LOS. The inherent weakness of the hard LOS criteria compelled us to develop fuzzy LOS criteria for transportation facilities or services. Therefore, we studied BRT fuzzy LOS criteria for the problem of passenger market segmentation in China.

\section{User Perceptions}

Transit passengers are heterogeneous and passengers' perceptions vary across different groups of passengers. The definition of LOS indicates that it needs to reflect user perceptions of quality of service provided by a facility or service. Thus, we conducted transit market segmentation based on user perceptions.

The journey of an individual taking a BRT bus includes traveling from the origin to the boarding station, waiting for a bus at the station, riding on the bus, and traveling from the alighting station to the destination. We selected a representative factor that influences user perceptions of quality of service in each subjourney. By doing so, user perceptions were defined as five-dimensional vectors of perceived arrival time, wait time, bus speed, passenger load, and departure time in this study. Perceived arrival time is the time taken by a passenger traveling from the origin to the boarding station as perceived by the passenger, which is different from the actual arrival time. Perceived wait time is the time a passenger spends waiting for a bus at a bus station according to his or her own perception, which differs from the actual wait time. Perceived bus speed is a passenger experience rating on the bus running speed while commuting in the bus. This is a categorical variable with a value of one expressing a rating of "very poor" and a value of five representing a rating of "very good." Perceived passenger load is a passenger experience rating on crowding encountered while riding on the bus, which is a categorical variable with a value of one expressing a rating of "very poor," and a value of five representing a rating of "very good." Note that in BRT passenger travel surveys, perceived bus speed and passenger load had a scale of one (very poor) to ten (very good). The ten-point scale was changed into a five-point scale in the analysis stage with the values one and two on the original scale integrated into the value one on the new scale. Similarly, the original values three and four were integrated into the value two and so on. The perceived departure time is the time taken by a passenger traveling from the alighting station to the destination according to his or her own perception, which is different from the actual departure time.

\section{Data Collection}

4.1. Transit Travel Survey System. We developed a smartphone-based transit travel survey system. This system consists of an app, a server, and a web interface. The app (Figure 1) interacts with users and collects and uploads the data to the server. The server stores the data and prepares them for the web interface. The web interface is the output 
end of the system, from which the collected data are downloaded.

The app covers the entire trip of a passenger using the transit system and can record the complete travel information. Personal demographics, time elapsed, longitudes, and latitudes, that users present at each travel node, and user perceptions, can all be recorded. All the recorded data above are transmitted in real-time to the server. The server computes travel times in each subjourney and the bus speed, using real-time algorithms. Furthermore, it obtains the ride distance by invoking the Baidu map program. The server stores all of the data and transmits them to the web interface.

The collected data by the transit travel survey system include personal demographics, time spent by users at each travel node, times that the users spend in each subjourney, user perceptions, and other travel information. In particular, personal demographics contain gender, age, education, occupation, income, car ownership status, travel mode, BRT use frequency, and user's city of habitation. The time elapsed from the moment that a user sets out from the origin, arrives at the boarding station, boards a bus, alights from the bus, and arrives at the destination is collected. Arrival time, wait time, in-vehicle time, and departure time are all gathered. User perceptions, i.e., perceived arrival time, wait time, bus speed, passenger load, and departure time, are gathered. Other travel information such as travel purpose, bus route number, ride distance, and bus speed is collected as well.

4.2. BRT Passenger Travel Surveys. The Guangzhou BRT was opened in 2010 and has an average daily ridership of more than 850,000 passengers, higher than most metro and all light rail lines worldwide. It won the sustainable transport award in 2011 and is the only gold standard BRT in Asia. The Changzhou BRT was opened in 2008; its daily ridership exceeds 310,000 passengers, which accounts for $30 \%$ of the total public transit ridership. It won the Tien-Yow Jeme Civil Engineering Prize in 2010, making it the first public transit project to have won this prize. The Yichang BRT opened in 2015 and serves over 240,000 riders a day. Twenty percent of BRT riders previously drove a car or took a taxi. The city of Yichang won the sustainable transport award in 2016. We selected the Guangzhou, Changzhou, and Yichang BRTs to conduct passenger travel surveys using the transit travel survey system. The basic survey process includes recruiting participants and training them to use our cellphone app to collect the relevant data during daily travel.

Recruiting participants was the most difficult but crucial step. We tried several strategies to gather participants, such as by recruitment via an investigative firm, enrollment from WeChat groups, and enlistment via recommendations from acquaintances. However, these strategies could only bring together a small number of samples. Finally, we tried out a scheme wherein participants were recruited face-to-face at stations and on buses. Our experience with this technique proves that face-to-face communication with passengers at stations or on buses is the most effective way of recruiting participants. Four of us, acting as surveyors, entered the stations and randomly looked for passengers to converse with one-on-one. To begin with, we introduced ourselves, described our purpose in talking to them, and explained the task we were engaged in. Next, we invited passengers to participate in the survey. If the passenger was willing to participate, we added him/her to WeChat. This is very important because we had to teach them how to use the app through WeChat. We send the app's two-dimensional code and the reward both through WeChat as well. Meanwhile, we encouraged him/her to immediately download and install the app (1-2 minutes to complete such an install) by scanning the app's two-dimensional code at the station. If he/she has not installed the app when the bus arrives, we sent the app's two-dimensional code by WeChat and guided the person through the installation later. The participants recruited by a single surveyor in one day comprise a WeChat group. We reminded participants of each such group to use the app in daily travel and solve common problems that are encountered. The disadvantage of the aforementioned strategy is that usually the WeChat id of the passengers is not added by the time the bus arrives. This resulted in much wasted effort. Therefore, we attempted to recruit participants on the bus itself. We selected a bus that traveled on a route within the BRT system and randomly invited passengers to participate in the survey. We had ample time to communicate with passengers on the bus. However, surveyors became exhausted and succumbed to motion sickness owing to the rough terrain that the bus traveled over. Surveyors often had no energy to move back and forth to look for passengers after getting two or three participants and therefore, rarely sought out participants on the bus.

Those stations having large passenger volumes were selected to recruit participants. In the case of the Guangzhou BRT, we surveyed the stations of Gangding, Normal University and Jinan University, Shipai Qiao, and Tangxiacun. We also took route B1. The four surveyors representing each of the stations mentioned above worked from November 4 to 6,2017 , and about 65 participants were added. In the case of the Changzhou BRT, the stations of Wanfu Qiao, Huaide Road, Yanling Road, Lanling Road, and Renmin Road and Qinggong College were surveyed, and 100 participants were recruited over the period ranging from November 26 to 29, 2017. In the case of the Yichang BRT, the working stations were Wuyi Square, Pagoda River, Liujiadayan, and Lvluo Road. and 100 participants were enlisted from January 16 to 19,2018 . In these three cities, 265 participants were recruited in total.

After finding participants who were interested in the survey, data collection began. Participants took part in the survey for data collection using our app during daily BRT travel. A single data record was received for every participant who successfully used the app on a BRT trip. The data collection continued for one month in each city. Over the duration of the survey, participants were free to decide whether to continue their participation. Surveyors frequently encouraged them to use the app when taking the BRT.

From the participants' point of view, participation in survey took place as follows: (1) Surveyors invite passengers to participate in the BRT passenger travel survey. (2) 


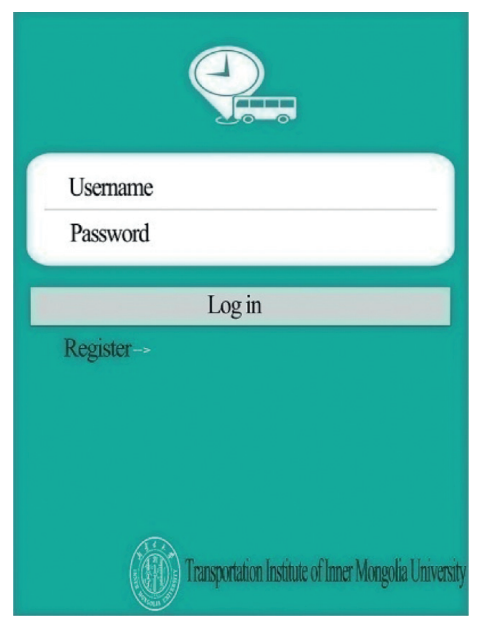

(a)

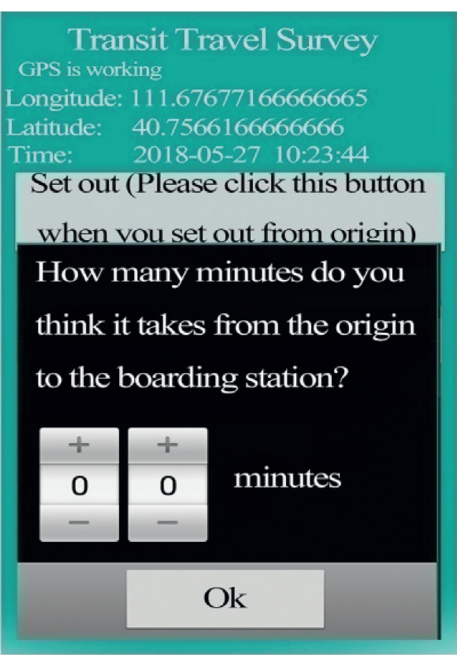

(d)

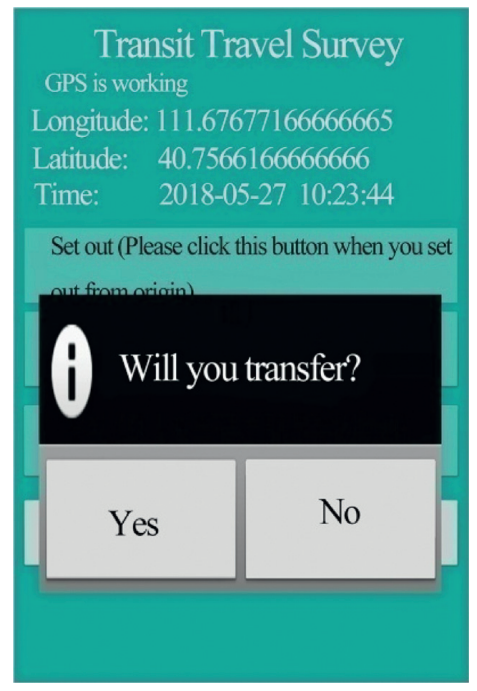

(g)

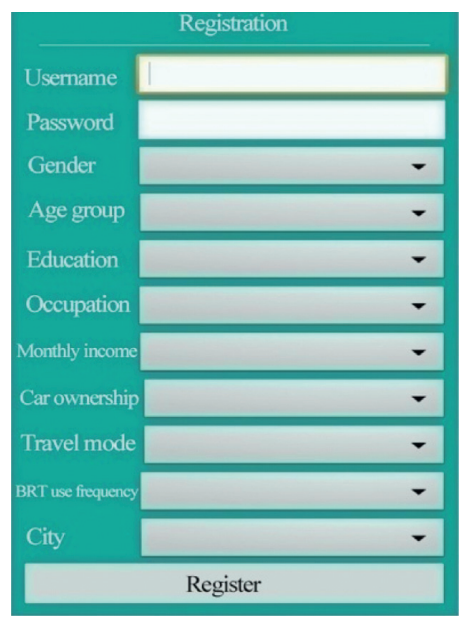

(b)

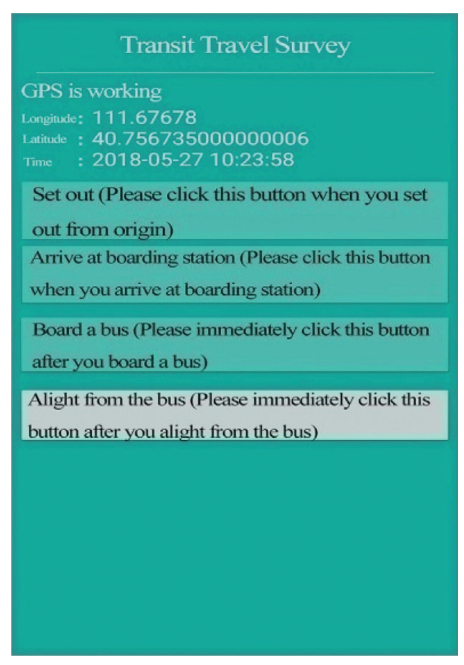

(e)

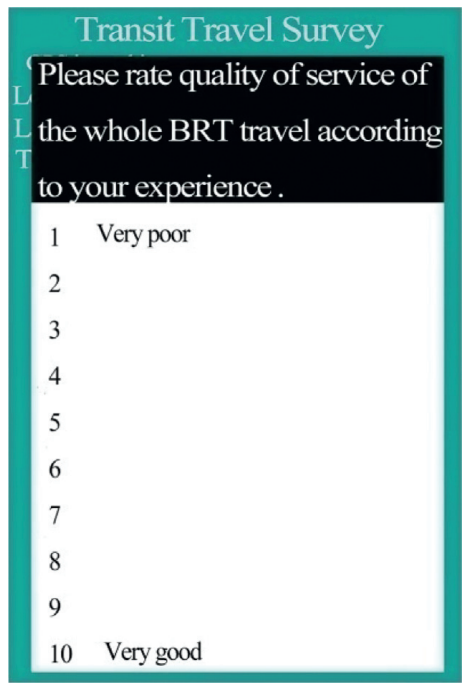

(h)

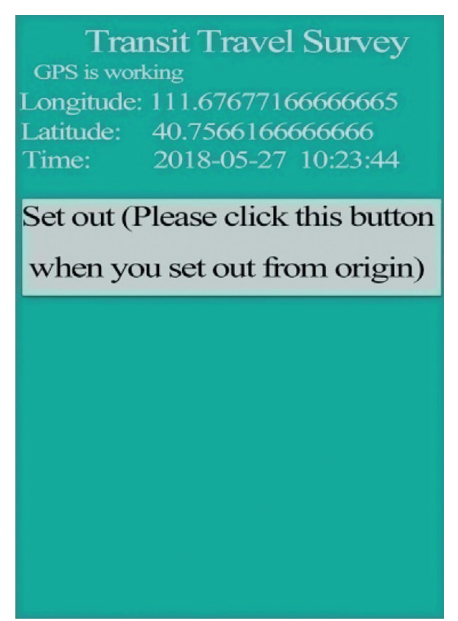

(c)

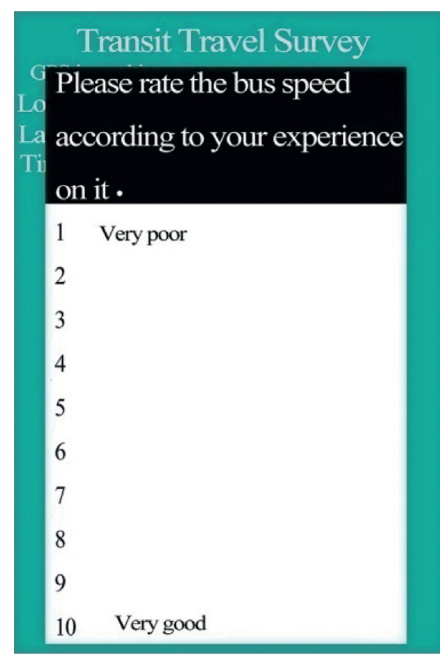

(f)

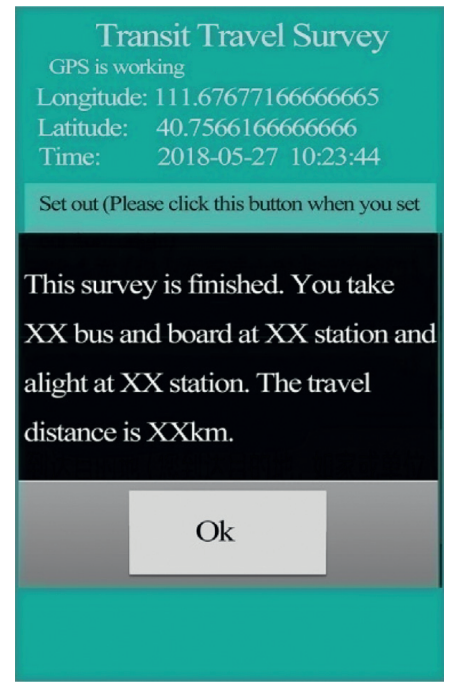

(i)

Figure 1: App interface (partial).

Passengers accept their invitation and become participants.

(3) Participants download and install the app and register.

(4) Prior to the formal survey, the participants take a pilot survey to be familiar with app. (5) During daily BRT travel, participants use the app over the entire trip to participate in the survey. They invoke the app and log in before they leave 
the originating point of their journey. They operate the app following the prompts at each of the travel nodes that comprise the entire trip. The app runs in the background when no operations are being performed. (6) After completing the BRT travel route (i.e., there is no more use for the app), participants take a screenshot of the final app interface and send the screenshot to surveyors. (7) Surveyors judge whether the participants have used the app truly and correctly (i.e., whether the collected data are valid) by studying this final interface. (8) After completing a valid survey (i.e., correctly using the app on a single BRT trip), participants receive a reward of $\$ 1.50$. Participants are free to end or continue their participation.

4.3. Sample Characteristics. The BRT passenger travel surveys produced a dataset with a sample size of 1304. Fiftyfive percent of the sample was male. Ninety-five percent of the participants in the sample fell in the 19-30 year old age group or in the 31-50 year old age group. Eighty-three percent of the sample held a degree from a technical college or a bachelor's degree. Different occupations were equally represented in the sample. Sixty-five percent of the participants in the sample had a monthly income lower than $\$ 780$. Sixty-three percent of the sample did not own a car. Most of the sample participants were frequent transit users. Among the participants in the sample, ninety-eight percent of them used the BRT for transit. Sixty-eight percent of the participants in the sample used the BRT every day. Sixtyseven percent of the sample stated that the purpose of travel was to commute, and twenty-five percent claimed recreation as the reason for travel. In general, the sample was, perhaps not surprisingly, composed mostly of young and middle-aged people, riders with a midlevel to upper level of education, low-income riders, riders without a car, and frequent transit users.

\section{Methodology}

We conducted transit market segmentation based on user perceptions. Because a user's perceived bus speed and passenger load are multivariate categorical data, we employed the latent class model to conduct BRT passenger segmentation.

Over the course of the entire journey of a passenger taking a BRT bus, the following factors influence user perceptions-passenger arrival time, wait time, bus speed, passenger departure time, facility condition (width, height, isolation, and cleanliness), availability of real-time bus arrival information, availability of shelter and benches, passenger load, driver attitude, and seating comfort. Of all the factors mentioned here, passengers most easily perceive those that are time related. Thus, passenger arrival time, passenger wait time, bus running speed, and passenger departure time were selected as service metrics to reflect BRT LOS. We developed fuzzy LOS criteria for BRT using fuzzy C-means clustering to deal with the ambiguity in the criteria.
5.1. Latent Class Model (LCM). The LCM seeks to stratify the cross-classification table of manifest variables by a latent variable that eliminates all confounding factors between the manifest variables [21]. The LCM approximates the observed joint distribution of the manifest variables as the weighted sum of a finite number $R$, denoting the number of latent classes, of constituent cross-classification tables [21]. The probability that an individual $i$ in class $r(r=1,2, \ldots, R)$ produces a particular set of outcomes on the manifest variables, assuming local independence, is the product in the following equation:

$$
f\left(Y_{i} ; \pi_{r}\right)=\prod_{j=1}^{J} \prod_{k=1}^{K_{j}}\left(\pi_{j r k}\right)^{Y_{i j k}},
$$

where $J$ denotes the number of manifest variables, $j=1,2, \ldots, J ; K_{j}$ denotes the number of categories (outcomes) of the $j$ th manifest variable, $k=1,2, \ldots, K_{j} ; Y_{i j k}$ denotes the observed values of the manifest variables such that $Y_{i j k}=1$ if an individual $i(i=1,2, \ldots, N)$ provides the $k$ th outcome to the $j$ th manifest variable, and $Y_{i j k}=0$ otherwise; $\pi_{j r k}$ denotes the conditional probability that an individual $i$ in class $r$ produces the $k$ th outcome on the $j$ th manifest variable. Within each class, for each manifest variable, $\sum_{k=1}^{K_{j}} \pi_{j r k}=1$.

The probability density function across all classes is the weighted sum in the following equation:

$$
P\left(Y_{i} \mid \pi, p\right)=\sum_{r=1}^{R} p_{r} \prod_{j=1}^{J} \prod_{k=1}^{K_{j}}\left(\pi_{j r k}\right)^{Y_{i j k}},
$$

where $p_{r}$ denotes the $R$ mixing proportions that provide the weights in the weighted sum of the component tables, with $\sum_{r} p_{r}=1$.

The parameters estimated by the LCM are $p_{r}$ and $\pi_{j r k}$. The LCM is estimated by maximizing the log-likelihood function in the following equation:

$$
\log L=\sum_{i=1}^{N} \ln \sum_{r=1}^{R} p_{r} \prod_{j=1}^{J} \prod_{k=1}^{K_{j}}\left(\pi_{j r k}\right)^{Y_{i j k}},
$$

with respect to $p_{r}$ and $\pi_{j r k}$; the expectation maximization (EM) algorithm is used for parameter estimation.

A priori, the number of the latent class $R$ is unknown. Therefore, the goal of model selection is to find the model that is parsimonious and fits better to the study data. Model selection is conducted by fitting a complete independence model with $R=1$ and iteratively increasing the number of latent class by one until a suitable fit has been achieved. The two most widely used criteria of model selection are the Bayesian information criterion (BIC) and the Akaike information criterion (AIC). Preferred models are those that minimize the values of the BIC and/or AIC [10].

5.2. Fuzzy C-Means Clustering (FCM). The FCM is a clustering algorithm that groups the data points in multidimensional space into a specific number of clusters. 
Furthermore, every data point in the dataset belongs to every cluster according to a certain membership [22]. The purpose of FCM is to determine the cluster center $V=\left(v_{1}, v_{2}, \ldots, v_{c}\right)$ and the associated membership matrix $U=\left\{u_{i j}\right\}$. It is based on the minimization of the objective function in

$$
J_{m}=\sum_{j=1}^{n} \sum_{i=1}^{c} \mu_{i j}^{m}\left\|x_{j}-v_{i}\right\|^{2}
$$

where $x_{j}$ is the $j$ th measured data point; $n$ is the number of data points; $v_{i}$ is the $i$ th cluster center; $c$ is the number of clusters; $u_{i j}$ is the membership of $x_{j}$ in cluster $i$; and $m$ is a fuzziness index.

Fuzzy partitioning is carried out by utilizing an iterative optimization of the objective function with an adjustment of membership $u_{i j}$ and cluster center $v_{i}$ by using the following equations [22]:

$$
\mu_{i j}=\frac{1}{\sum_{q=1}^{c}\left(\left\|x_{j}-v_{i}\right\| /\left\|x_{j}-v_{q}\right\|\right)^{2 /(m-1)}}, \quad 1 \leq i \leq c, 1 \leq j \leq n,
$$

$$
v_{i}=\frac{\sum_{j=1}^{n} \mu_{i j}^{m} x_{j}}{\sum_{j=1}^{n} \mu_{i j}^{m}}, \quad 1 \leq i \leq c .
$$

This iteration will stop when $\max _{i j}\left(\left|u_{i j}^{(k+1)}-u_{i j}^{k}\right|\right)<\varepsilon$, where $\varepsilon$ is a termination criterion.

\section{Results}

6.1. Results of Transit Market Segmentation. Based on the user perceptions data collected from BRT passenger travel surveys, we carried out transit market segmentation using LCM. Transit market segmentation aims to discover differentiated passenger groups according to perception or psychological factors. An assessment of whether the stratified passenger groups are significantly different was carried out based on the conditional probabilities $\left(\pi_{j r k}\right)$ in LCM. In this research work, user perceptions include the perceived arrival time, wait time, bus speed, passenger load, and departure time. Perceived arrival, wait, and departure time are continuous variables and need to be converted into categorical variables.

Initially, all of these types of user perceptions were assumed to be manifest variables of the LCM to stratify passengers. However, the passenger groups stratified by assigning latent class number from one to ten exhibited no significant differences from one another. We also tried to combine some types of user perceptions as manifest variables and still did not find significantly different passenger groups. Next, all or some of these types of user perceptions were used as manifest variables, and passenger social-demographics such as gender, age, and education were used as covariates; latent class regression models were adopted to classify passengers; however, again, no different passenger groups were identified.

We created two new variables known as the class arrival time ratio and the class wait time ratio. The arrival time ratio is defined as perceived arrival time divided by actual arrival time. The class arrival time ratio is defined as a categorical variable; it assumes a value of unity if the arrival time ratio is smaller than, or equal to one, and it becomes two if the arrival time ratio is greater than one. The wait time ratio is defined as the perceived wait time divided by the actual wait time. The class wait time ratio is defined as a categorical variable that assumes a value of unity if the wait time ratio is smaller than or equal to one and a value of two if the wait time ratio is bigger than one.

Using the aforementioned two new variables along with perceived bus speed, passenger load, and departure time as manifest variables and passenger sociodemographics as covariates, latent class regression models were developed. Various combinations of these variables to stratify passengers did not lead to the discovery of significantly different passenger groups.

By gradually changing the manifest variables and the number of latent classes, distinct passenger groups were finally discovered. Using the class arrival time ratio, class wait time ratio, and perceived bus speed as manifest variables, models with latent classes of one to four were developed and the significantly different passenger groups were identified. The values of AIC and BIC for models with one to four classes were 5563.84 and 5593.29, 5505.49 and 5569.29, 5512.34 and 5610.50, and 5523.34 and 5655.85. The values of AIC and BIC for the two-class model were minimal, and thus, this model was adjudged the most appropriate and parsimonious model. Therefore, we stratified BRT passengers into two groups based on the accuracy of the fit results of LCM. The results of the estimated conditional probabilities and class probabilities of the two-class model are shown in Table 1.

Group 1 is composed of $60 \%$ of the sample data. All members of group $1(100 \%)$ are passengers whose wait time ratio $\leq 1$ (perceived wait time $\leq$ wait time), $57 \%$ of the passengers are those whose arrival time ratio $\leq 1$ (perceived arrival time $\leq$ arrival time), and $66 \%$ of the passengers are those whose perceived bus speed is good. Group 2 consists of $40 \%$ of the sample data. In group 2, $90 \%$ of the passengers are those whose wait time ratio $>1$ (perceived wait time $>$ wait time), 62\% of the passengers are those whose arrival time ratio $>1$ (perceived arrival time $>$ arrival time), and $62 \%$ of the passengers are those whose perceived bus speed is good. Passengers who are in a hurry and anxious to catch the bus always perceive wait time or arrival time to be longer than their actual values. On the contrary, passengers who are not in a hurry and are calm and relaxed may perceive wait time or arrival time to be shorter than their actual values. Therefore, group 1 was called the calm passenger group and group 2, the anxious passenger group. The calm passenger group (group 1) perceives bus speed to be slightly better than the anxious passenger group (group 2).

6.2. Results for Fuzzy LOS Criteria. Based on the service metrics (passenger arrival time, passenger wait time, and bus running speed), data collected from BRT passenger 
TABLE 1: Estimated conditional and class probabilities with two latent classes.

\begin{tabular}{lccc}
\hline Manifest variables & Categories & Group 1 & Group 2 \\
\hline \multirow{2}{*}{ Class arrival time ratio } & 1 (arrival time ratio $\leq 1)$ & 0.57 & 0.43 \\
& 2 (arrival time ratio $>1)$ & 1.00 & 0.62 \\
\hline \multirow{2}{*}{ Class wait time ratio } & 1 (wait time ratio $\leq 1)$ & 0.00 & 0.10 \\
& 2 (wait time ratio $>1)$ & 0.12 & 0.90 \\
\hline & 1 (very poor) & 0.00 & 0.11 \\
Perceived bus speed & 2 (poor) & 0.22 & 0.09 \\
& 3 (neutral) & 0.38 & 0.32 \\
\hline Estimated class probabilities & 4 (good) & 0.28 & 0.30 \\
\hline
\end{tabular}

travel surveys and BRT fuzzy LOS criteria for the calm and the anxious passenger groups were developed using FCM. Initially, these three service metrics were divided into six clusters for each passenger group using FCM, following which, cluster centers and membership matrices were obtained. Next, membership functions for each service metric for each passenger group were derived based on the membership matrices. Subsequently, fuzzy LOS criteria for these three service metrics for each passenger group were developed by analyzing the membership functions.

Figure 2 shows the membership functions for the calm passenger group. Figures 2(a), 2(c), and 2(e) are the original membership functions of passenger arrival time, passenger wait time, and bus running speed, respectively. Figures 2(b), 2(d), and 2(f)are their respective approximated membership functions. The six different curves in each figure represent six different clusters, i.e., six levels of service, $A-F$. Figure 3 depicts the membership functions for the anxious passenger group, which include the original and approximated membership functions of passenger arrival time, passenger wait time, and bus running speed. Membership functions for passenger departure time are almost the same as those for the passenger arrival time; therefore, they have not been shown herein.

Tables 2 and 3 show BRT fuzzy LOS criteria for the calm passenger group, in which Table 2 displays ranges and centers of service metrics for fuzzy LOS categories, $A-F$, Table 3 displays ranges of service metrics for a single primary LOS category (membership value $>0.5$ ) and a single secondary LOS category (membership value $<0.5$ ). Tables 4 and 5 show BRT fuzzy LOS criteria for the anxious passenger group, which includes the ranges and centers for fuzzy LOS categories (Table 4) and the ranges for one primary category and one secondary LOS category ( Table 5). As observed from Tables 2-5, for each fuzzy LOS category $A-F$, the boundary values of the passenger wait time for the anxious passenger group are smaller than those for the calm passenger group. Furthermore, the boundary values of the bus running speed for the anxious passenger group are higher than those for the calm passenger group. This result matches with the psychological characteristics of the two passenger groups. For example, the upper boundary values of the passenger wait time for the anxious passenger group are 2.4, 4.4, 7.3, 12.6, and 19.3, respectively, while the corresponding values for the calm passenger group are 2.9, 5.4, 8.5, 13.2, and 23.1. The lower boundary values of the bus running speed for the anxious passenger group are 27.4, 21.8, 18.1, 14.9, 10.8, and zero, respectively, while the corresponding values for the calm passenger group are 24.9, 20.9, 17.4, 14.2, 10.4, and zero. Anxious passengers are always in a hurry and have higher anticipations of BRT service quality, i.e., they expect shorter wait times and higher bus speeds. By contrast, calm passengers are more tolerant and are able to accept longer wait times and slower bus speeds. Therefore, exactly as shown in Tables 2-5, anxious passenger groups have smaller boundary values of passenger wait time and higher boundary values of bus running speed than those of the calm passenger groups.

LOS criteria in the TCQSMs or HCMs use clear-cut boundaries for separating each LOS category, wherein a certain value of service metric exclusively belongs to one LOS category, which is called the hard LOS criteria in this study. An inherent weakness of the hard LOS criteria is that some small changes in service metrics can appear significant, while some big changes in service metrics can appear trivial, which limits the accuracy with which transportation facilities or services may be characterized $[11,12]$. Specifically, the LOS changes from one category to the other when service metric values near the boundaries generate a small variation, while the LOS remains unchanged when service metric values display a large variation from the lower to the upper boundaries. For example, Das and Pandit established hard LOS criteria for passenger wait time for bus transit in India, where the ranges for LOS' $A, B, C, D$, and $E$, respectively, are $0 \mathrm{~min}$, 0.1-4.0 $\mathrm{min}, 4.1-20.0 \mathrm{~min}, 20.1-35.0 \mathrm{~min}$, and $>35.0 \mathrm{~min}$. The values of $0 \mathrm{~min}, 4.0 \mathrm{~min}, 20.0 \mathrm{~min}$, and $35.0 \mathrm{~min}$ are the clear-cut boundaries for separating LOS' $A, B, C, D$, and $E$. The LOS changes from $B$ to $C$ when passenger wait time varies from $4.0 \mathrm{~min}$ to $4.1 \mathrm{~min}$, while the LOS remains at $C$ when passenger wait time varies from $4.1 \mathrm{~min}$ to $20.0 \mathrm{~min}$ [23]. As observed from Tables 2-5, fuzzy LOS criteria replace clear boundaries with fuzzy boundaries, i.e., a certain degree of overlap between adjacent LOS categories exists. Furthermore, the service metric values in the overlapping sections belong to adjacent LOS categories with varying memberships. The values of the memberships are derived from membership functions (Figures 2 and 3). 


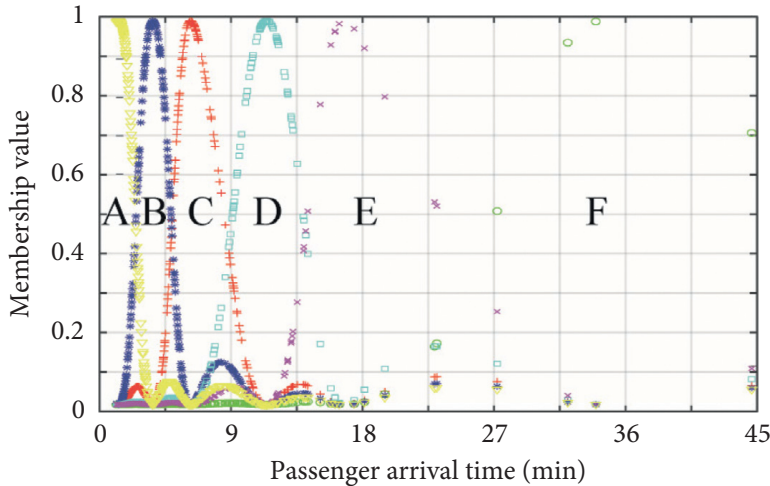

(a)

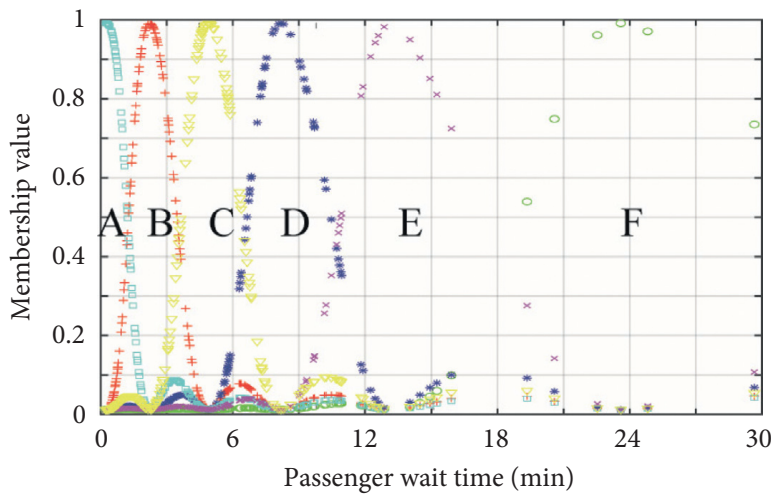

(c)

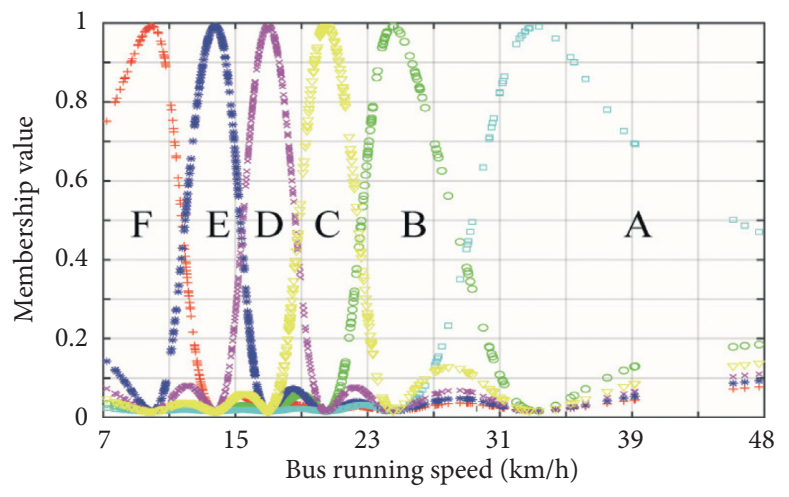

(e)

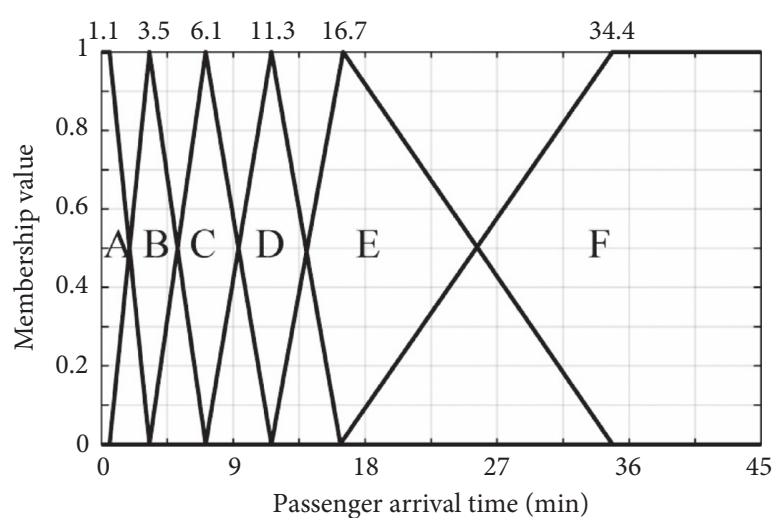

(b)

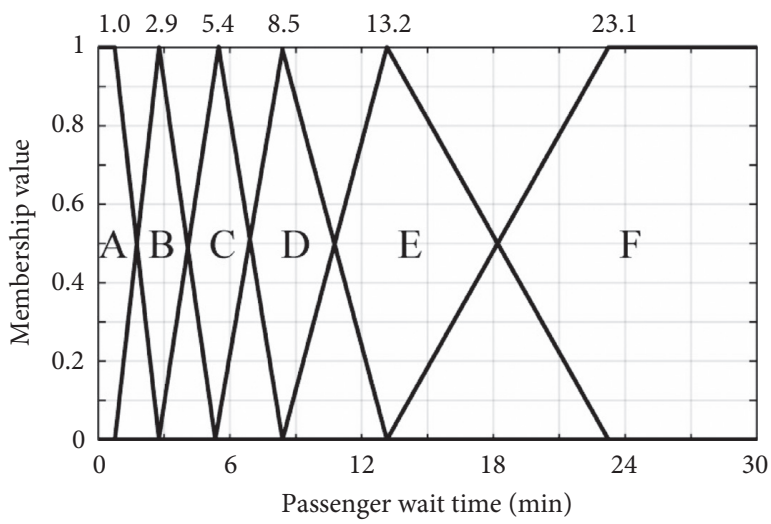

(d)

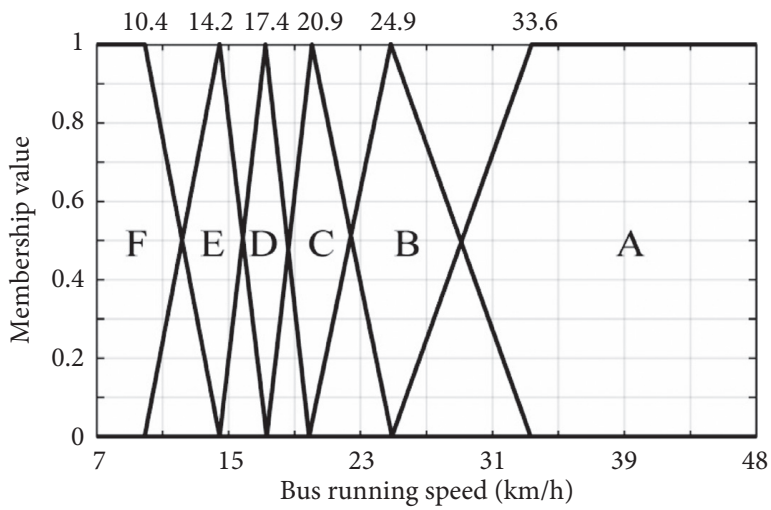

(f)

Figure 2: Membership functions for the calm passenger group. (a, c, and e) The original membership functions; (b, d, and f) the approximated membership functions.

Consider the fuzzy LOS criteria of passenger wait time for the anxious passenger group (the second row in Table 4) as an example. The sections of $1.2-2.4 \mathrm{~min}, 2.4-4.4 \mathrm{~min}$, 4.4-7.3 $\mathrm{min}, 7.3-12.6 \mathrm{~min}$, and $12.6-19.3 \mathrm{~min}$ are the overlapping sections of LOS' $A$ and $B, B$ and $C, C$ and $D, D$ and $E$, and $E$ and $F$, respectively. The values of passenger wait time in these overlapping sections belong to adjacent LOS categories with different memberships as shown in Figure 3(d). The value of $4.0 \mathrm{~min}$ belongs to LOS' $B$ and $C$ with memberships of 0.1 and 0.9 . The value of $4.1 \mathrm{~min}$ belongs to LOS' $B$ and $C$ with memberships of 0.05 and
0.95. That is, the values of $4.0 \mathrm{~min}$ and $4.1 \mathrm{~min}$ belong to LOS' $B$ and $C$ simultaneously with different memberships, rather than being exclusively classified into $B$ or $C$ as in the hard LOS criteria. Fuzzy LOS criteria can accurately describe the extent to which service metric values belong to the adjacent LOS categories by the use of membership. Furthermore, the continuous changes in membership can reflect the LOS changes resulting from variations in the service metric values, thus permitting fuzzy LOS criteria to effectively overcome the weakness inherent in the hard LOS criteria. 


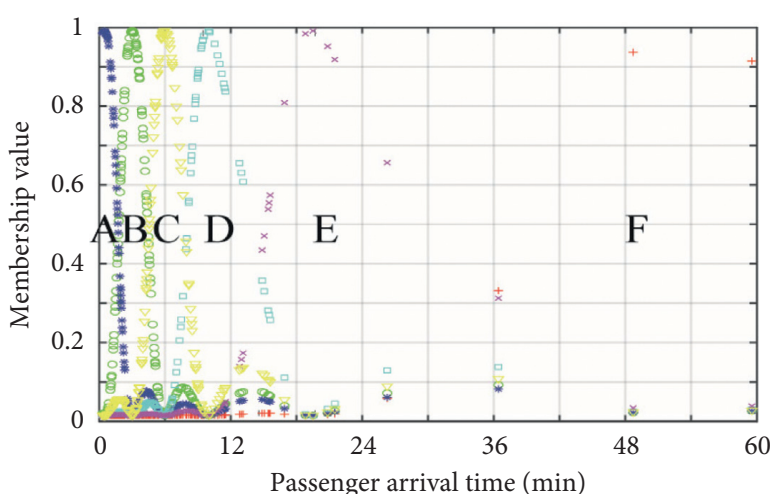

(a)

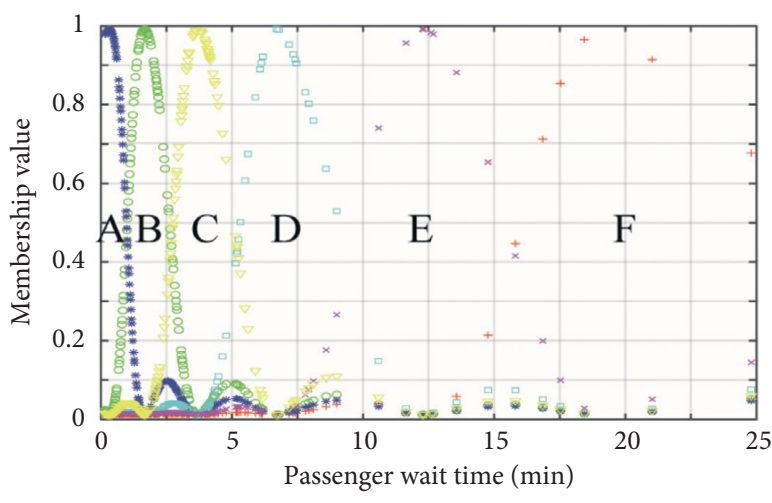

(c)

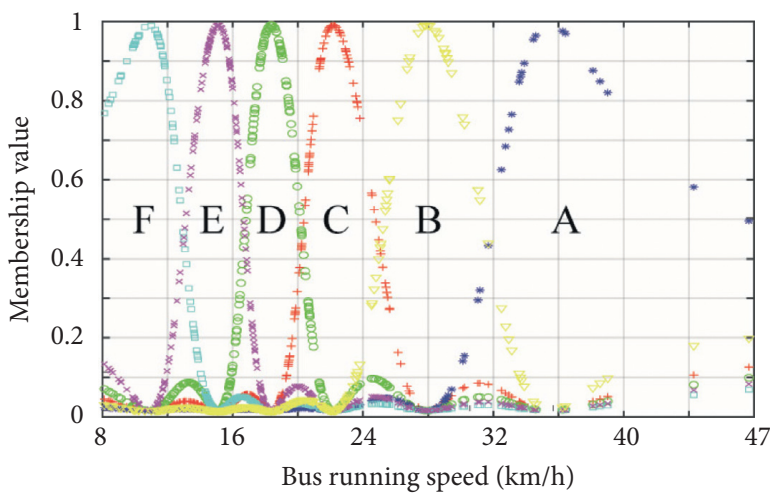

(e)

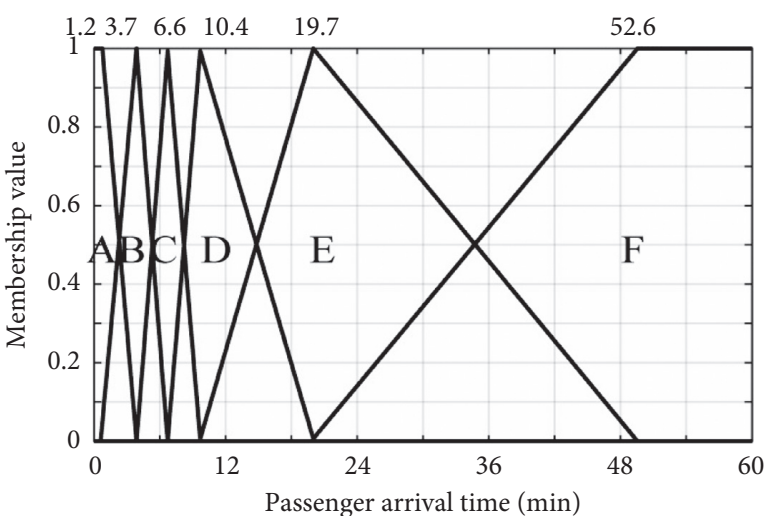

(b)

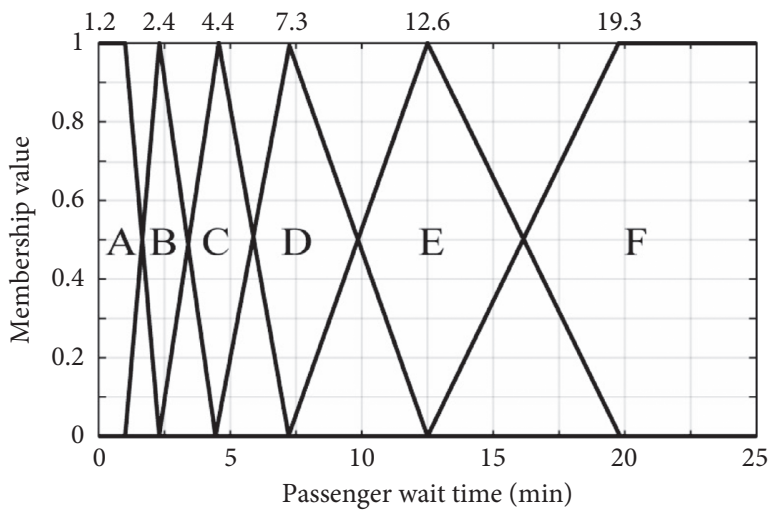

(d)

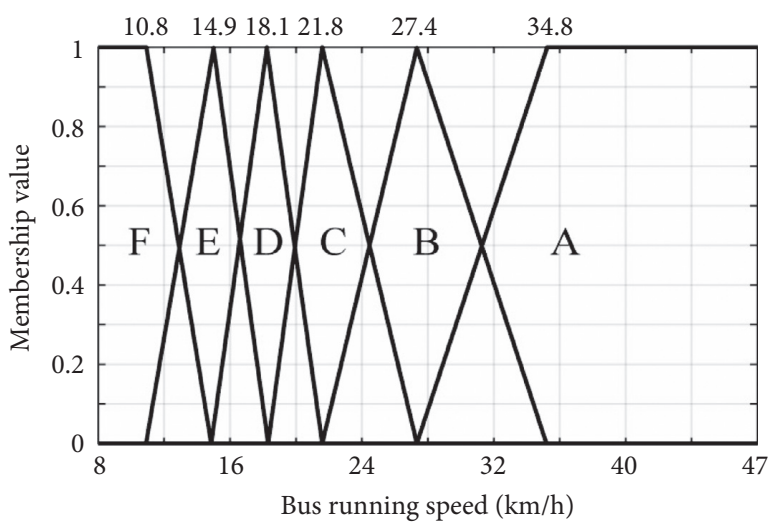

(f)

Figure 3: Membership functions for the anxious passenger group (a, c, and e) The original membership functions; (b, d, and f) the approximated membership functions).

TABLE 2: BRT fuzzy LOS criteria for the calm passenger group (ranges and centers of service metrics for fuzzy LOS categories).

\begin{tabular}{|c|c|c|c|c|c|c|c|}
\hline \multirow{2}{*}{ Service metrics } & & \multicolumn{6}{|c|}{ Fuzzy LOS categories } \\
\hline & & $A$ & $B$ & C & $D$ & $E$ & $F$ \\
\hline Passenger arrival time (min) & $\begin{array}{l}\text { Center } \\
\text { Range }\end{array}$ & $\begin{array}{c}1.1 \\
(0,3.5)\end{array}$ & $\begin{array}{c}3.5 \\
(1.1,6.1)\end{array}$ & $\begin{array}{c}6.1 \\
(3.5,11.3) \\
\end{array}$ & $\begin{array}{c}11.3 \\
(6.1,16.7)\end{array}$ & $\begin{array}{c}16.7 \\
(11.3,34.4)\end{array}$ & $\begin{array}{c}34.4 \\
(16.7, \infty)\end{array}$ \\
\hline Passenger wait time (min) & $\begin{array}{l}\text { Center } \\
\text { Range }\end{array}$ & $\begin{array}{c}1.0 \\
(0,2.9)\end{array}$ & $\begin{array}{c}2.9 \\
(1.0,5.4)\end{array}$ & $\begin{array}{c}5.4 \\
(2.9,8.5)\end{array}$ & $\begin{array}{c}8.5 \\
(5.4,13.2)\end{array}$ & $\begin{array}{c}13.2 \\
(8.5,23.1)\end{array}$ & $\begin{array}{c}23.1 \\
(13.2, \infty)\end{array}$ \\
\hline Bus running speed $(\mathrm{km} / \mathrm{h})$ & $\begin{array}{l}\text { Center } \\
\text { Range }\end{array}$ & $\begin{array}{c}33.6 \\
(24.9, \infty)\end{array}$ & $\begin{array}{c}24.9 \\
(20.9,33.6)\end{array}$ & $\begin{array}{c}20.9 \\
(17.4,24.9)\end{array}$ & $\begin{array}{c}17.4 \\
(14.2,20.9)\end{array}$ & $\begin{array}{c}14.2 \\
(10.4,17.4)\end{array}$ & $\begin{array}{c}10.4 \\
(0,14.2)\end{array}$ \\
\hline
\end{tabular}


TABLE 3: BRT fuzzy LOS criteria for the calm passenger group (ranges of service metrics for one primary and one secondary LOS category).

\begin{tabular}{|c|c|c|c|c|}
\hline \multicolumn{2}{|c|}{$\begin{array}{l}\text { Membership for fuzzy } \\
\text { LOS categories }\end{array}$} & \multicolumn{3}{|c|}{$\begin{array}{c}\text { Service metrics } \\
\text { Passenger wait time (min) }\end{array}$} \\
\hline$A>0.5$ & $B<0.5$ & $(0,2.3)$ & $(0,2.0)$ & $(29.3, \infty)$ \\
\hline \multirow{2}{*}{$B>0.5$} & $A<0.5$ & $(2.3,3.5)$ & $(2.0,2.9)$ & $(24.9,29.3)$ \\
\hline & $C<0.5$ & $(3.5,4.8)$ & $(2.9,4.2)$ & $(22.9,24.9)$ \\
\hline \multirow{2}{*}{$C>0.5$} & $B<0.5$ & $(4.8,6.1)$ & $(4.2,5.4)$ & $(20.9,22.9)$ \\
\hline & $D<0.5$ & $(6.1,8.7)$ & $(5.4,7.0)$ & $(19.2,20.9)$ \\
\hline \multirow{2}{*}{$D>0.5$} & $C<0.5$ & $(8.7,11.3)$ & $(7.0,8.5)$ & $(17.4,19.2)$ \\
\hline & $E<0.5$ & $(11.3,14.0)$ & $(8.5,10.9)$ & $(15.8,17.4)$ \\
\hline \multirow{2}{*}{$E>0.5$} & $D<0.5$ & $(14.0,16.7)$ & $(10.9,13.2)$ & $(14.2,15.8)$ \\
\hline & $F<0.5$ & $(16.7,25.6)$ & $(13.2,18.2)$ & $(12.3,14.2)$ \\
\hline$F>0.5$ & $E<0.5$ & $(25.6, \infty)$ & $(18.2, \infty)$ & $(0,12.3)$ \\
\hline
\end{tabular}

TABLE 4: BRT fuzzy LOS criteria for the anxious passenger group (ranges and centers of service metrics for fuzzy LOS categories).

\begin{tabular}{lccccccc}
\hline Service metrics & \multicolumn{5}{c}{ Fuzzy LOS categories } \\
& & $A$ & $B$ & $C$ & $D$ & $E$ & \multicolumn{3}{c}{$F$} \\
\hline \multirow{2}{*}{ Passenger arrival time $(\mathrm{min})$} & Center & 1.2 & 3.7 & 6.6 & 10.4 & 19.7 & 52.6 \\
& Range & $(0,3.7)$ & $(1.2,6.6)$ & $(3.7,10.4)$ & $(6.6,19.7)$ & $(10.4,52.6)$ & $(19.7, \infty)$ \\
\hline \multirow{2}{*}{ Passenger wait time $(\mathrm{min})$} & Center & 1.2 & 2.4 & 4.4 & 7.3 & 12.6 & 19.3 \\
& Range & $(0,2.4)$ & $(1.2,4.4)$ & $(2.4,7.3)$ & $(4.4,12.6)$ & $(7.3,19.3)$ & $(12.6, \infty)$ \\
\hline \multirow{2}{*}{ Bus running speed $(\mathrm{km} / \mathrm{h})$} & Center & 34.8 & 27.4 & 21.8 & 18.1 & 14.9 & 10.8 \\
& Range & $(27.4, \infty)$ & $(21.8,34.8)$ & $(18.1,27.4)$ & $(14.9,21.8)$ & $(10.8,18.1)$ & $(0,14.9)$ \\
\hline
\end{tabular}

TABLE 5: BRT fuzzy LOS criteria for the anxious passenger group (ranges of service metrics for one primary and one secondary LOS category).

\begin{tabular}{|c|c|c|c|c|}
\hline \multicolumn{2}{|c|}{$\begin{array}{l}\text { Membership for fuzzy } \\
\text { LOS categories }\end{array}$} & \multicolumn{3}{|c|}{ Service metrics } \\
\hline$A>0.5$ & $B<0.5$ & $(0,2.5)$ & $(0,1.8)$ & $(31.1, \infty)$ \\
\hline$B>0.5$ & $\begin{array}{l}A<0.5 \\
C<0.5\end{array}$ & $\begin{array}{l}(2.3,3.7) \\
(3.7,5.2)\end{array}$ & $\begin{array}{l}(1.8,2.4) \\
(2.4,3.4)\end{array}$ & $\begin{array}{l}(27.4,31.1) \\
(24.6,27.4)\end{array}$ \\
\hline$C>0.5$ & $\begin{array}{l}B<0.5 \\
D<0.5\end{array}$ & $\begin{array}{l}(5.2,6.6) \\
(6.6,8.5)\end{array}$ & $\begin{array}{l}(3.4,4.4) \\
(4.4,5.9)\end{array}$ & $\begin{array}{l}(21.8,24.6) \\
(20.0,21.8)\end{array}$ \\
\hline$D>0.5$ & $\begin{array}{l}C<0.5 \\
E<0.5\end{array}$ & $\begin{array}{l}(8.5,10.4) \\
(10.4,15.1)\end{array}$ & $\begin{array}{l}(5.9,7.3) \\
(7.3,10.0)\end{array}$ & $\begin{array}{l}(18.1,20.0) \\
(16.5,18.1)\end{array}$ \\
\hline$E>0.5$ & $\begin{array}{l}D<0.5 \\
F<0.5\end{array}$ & $\begin{array}{l}(15.1,19.7) \\
(19.7,36.2)\end{array}$ & $\begin{array}{l}(10.0,12.6) \\
(12.6,16.0)\end{array}$ & $\begin{array}{l}(14.9,16.5) \\
(12.9,14.9)\end{array}$ \\
\hline$F>0.5$ & $E<0.5$ & $(36.2, \infty)$ & $(16.0, \infty)$ & $(0,12.9)$ \\
\hline
\end{tabular}

\section{Conclusions}

This study presents a framework to develop fuzzy LOS criteria for bus transit considering passengers' heterogeneity. Further, it proposes BRT fuzzy LOS criteria for different passenger groups in China. A smartphone-based transit travel survey system was created, with which BRT passenger travel surveys were conducted in Guangzhou, Changzhou, and Yichang, and the data for user perceptions and service metrics were collected. Transit market segmentation was performed based on user perceptions, and passengers were segmented into the calm passenger group and the anxious passenger group using the LCM. Passenger arrival time, passenger wait time, and bus running speed were selected as service metrics to reflect BRT LOS. BRT fuzzy LOS criteria for the calm passenger group and the anxious passenger group, respectively, were proposed using fuzzy C-means clustering.
The TCQSMs did not consider passengers' heterogeneity and provided a set of general LOS criteria for bus transit. However, this research work examines heterogeneity by passenger market segmentation and proposes BRT LOS criteria for anxious and calm passenger groups. It was discovered that the anxious passenger group had lower boundary values of passenger wait time and higher boundary values of bus running speed than those of the calm passenger group, which fits the psychological characteristics of the two groups. Simultaneously, it demonstrates that the LOS criteria in this study can better reflect the personalized travel needs of certain passenger groups.

The TCQSMs provided hard LOS criteria for bus transit. An inherent weakness of the hard LOS criteria is that some small changes in service metrics can result in a change of LOS, while some significant changes in service metrics may not trigger such changes. However, this study proposes fuzzy LOS criteria for BRT in China. Fuzzy LOS criteria can 
properly describe the extent to which service metric values belong to the adjacent LOS categories via the use of membership, so that they can overcome the inherent weakness of the hard LOS criteria.

It was quite difficult to recruit participants owing to the requirement that the transit travel survey system app has to be installed on the participants' handheld device. Therefore, the sample size used in this study was small. This is one limitation in the exploration of potential differences in perceptions between different cities. Our study focused on the heterogeneity in passenger perception. In the future, the heterogeneity both in passenger behavior and perception will be studied to conduct transit market segmentation.

\section{Data Availability}

The data used to support the findings of this study are available in supplementary information files.

\section{Conflicts of Interest}

The authors declare that there are no conflicts of interest regarding the publication of this research work.

\section{Acknowledgments}

This study was supported by the National Natural Science Foundation of China (No. 51668048). The authors deeply appreciate the support.

\section{Supplementary Materials}

The supplementary descriptions for the supplementary files. (1) The descriptions for the file of mydata.csv. The variables in the file of mydata.csv include gender, age, education, occupation, income, car, travel_mode, brt_freq, travel_purpose, arrival_time, arrival_time_p, wait_time, wait_time_p, speed_p, crowd_p, departure_time, departure_time_p, overall_p, young, middle_age, high_school, university, student, worker, low_income, middle_high_income, work, home, recreation, arrival_time_ratio, class_arrival_time_ratio, wait_time_ratio, and class_wait_time_ratio. The introductions for each variable are as follows. For gender, " 0 " represents female and " 1 " represents male. For age, "1" represents teenager and " 2 " represents middle-aged person. For education, “1” represents high school and below and " 2 " represents higher education. For occupation, " 1 ” represents self-employed, " 2 " represents other, " 3 " represents enterprise staff, " 4 " represents students, “5” represents unemployed persons, and " 6 " represents civil servant. For income, " 1 " represents under 3000 yuan, “2" represents 3000-5000 yuan, “3” represents 5000-7000 yuan, and " 4 " represents over 7000 yuan. For car, it refers to if there is a private car, " 0 " represents "no," and " 1 " represents "yes." For travel_mode, " 1 " represents transit and " 0 " represents other. For brt_freq, it means use frequency of BRT, "1" represents occasional ride, " 2 " represents 1-2 days per week, " 3 " represents 3-4 days per week, "4" represents weekdays, and "5" represents every day. For travel_purpose, "1" represents work travel, "2" represents going home, and “ 3 " represents other. arrival_time means the time a passenger traveling from origin to boarding station. arrival_time_p, which is the perceived arrival time, is the time a passenger travels from the origin to the boarding station perceived from his own perspective. wait_time is the time a passenger spends in waiting at a bus at the station. wait_time_p is the perceived waiting time, which is the time a passenger spends in waiting for a bus at the station perceived by himself. speed_p is the bus speed perception, which is a passenger's experience rating on bus running speed generated during riding on the bus on a scale of 1 to 10 with 1 representing very poor and 10 representing very good. crowd_p is the passenger load perception, which is a passenger experience rating on crowding encountered during riding on the bus on a scale of 1 to 10 with 1 representing very poor and 10 representing very good. departure_time is the time a passenger traveling from alighting station to destination. departure_time_p is the perceived departure time, which is the time a passenger travels from a lighting station to the destination perceived by himself. overall_p means a passenger experience rating on operational condition of the whole bus travel from the origin to the destination on a scale of 1 to 10 with 1 representing very poor and 10 representing very good. Young means if a passenger is a teenager, " 1 " represents "yes" and "0" represents "no." middle_age means that if a passenger is a middle-aged person, " 1 " represents "yes" and "0" represents "no." high_school means if the education level of a passenger is high school, " 1 " represents "yes" and "0" represents "no." University means if a passenger received higher education, "1" represents "yes" and " 0 " represents "no." Student means if a passenger is a student, "1" represents "yes" and "0" represents "no." Worker means if a passenger is a worker, " 1 " represents "yes" and "0" represents "no." low_income means if a passenger belongs to low-income group, “1” represents "yes" and "0" represents "no." middle_high_income means if a passenger belongs to middle- or high-income group, "1" represents "yes" and "0" represents "no." Work means if travel purpose is work travel, " 1 " represents "yes" and "0" represents "no." Home means if travel purpose is going home, " 1 " represents "yes" and "0" represents "no." Recreation means if travel purpose is recreation, " 1 " represents "yes" and "0" represents "no." Arrival_time_ratio (i.e., arrival time ratio) is defined as the perceived arrival time divided by arrival time. Class_arrival_time_ratio (i.e., class arrival time ratio) is defined as a categorical variable with it being 1 if arrival time ratio is smaller than or equal to 1 and it being 2 if arrival time ratio is bigger than 1 . Wait_time_ratio (i.e., wait time ratio) is defined as the perceived wait time divided by wait time. Class_wait_time_ratio (i.e., class wait time ratio) is defined as a categorical variable with it being 1 if wait time ratio is smaller than or equal to 1 and it being 2 if wait time ratio is bigger than 1 . (2) The descriptions for the file of mydata_class.csv. Comparde with the file of mydata.csv, the file of mydata_class.csv adds a variable of "class." The variables in the file of mydata_class.csv include gender, 
age, education, occupation, income, car, travel_mode, brt_freq, travel_purpose, arrival_time, arrival_time_p, wait_time, wait_time_p, speed_p, crowd_p, departure_time, departure_time_p, overall_p, young, middle_age, high_school, university, student, worker, low_income, middle_high_income, work, home, recreation, arrival_time_ratio, class_arrival_time_ratio, wait_time_ratio, class_wait_time_ratio, and class. For the same variables in these two files, they have the same meanings. BRT passengers are stratified into two classes using the latent class model in this paper. The variable of "class" represents the two stratified classes with " 1 " and " 2 ." (Supplementary Materials)

\section{References}

[1] Transportation Research Board of the National Academies, Highway Capacity Manual, Transportation Research Board, Washington, DC, USA, 2000.

[2] Transportation Research Board of the National Academies, Highway Capacity Manual, Transportation Research Board, Washington, DC, USA, 2010.

[3] K. Choocharukul, K. C. Sinha, and F. L. Mannering, "User perceptions and engineering definitions of highway level of service: an exploratory statistical comparison," Transportation Research Part A: Policy and Practice, vol. 38, no. 9-10, pp. 677-689, 2004.

[4] Transportation Research Board of the National Academies, "Transit capacity and quality of service manual," Transportation Research Board, Washington, DC, USA, TCRP Report 100, 2nd edition, 2003.

[5] Transportation Research Board of the National Academies, "Transit capacity and quality of service manual," Transportation Research Board, Washington, DC, USA, TCRP Report 165, 3rd edition, 2013.

[6] R. Cervero and C. D. Kang, "Bus rapid transit impacts on land uses and land values in seoul, Korea," Transport Policy, vol. 18, no. 1, pp. 102-116, 2011.

[7] Bus rapid transit-centre of excellence," 2019, http://www.brt. $\mathrm{cl} /$.

[8] L. M. Kieu, A. Bhaskar, and E. Chung, "Passenger segmentation using smart card data," IEEE Transactions on Intelligent Transportation Systems, vol. 16, no. 3, pp. 1537-1548, 2015.

[9] L. M. Kieu, Y. Ou, and C. Cai, "Large-scale transit market segmentation with spatial-behavioral features," Transportation Research Part C: Emerging Technologies, vol. 90, pp. 97-113, 2018.

[10] R. D. Oña, G. López, F. J. D. D. L. Rios, and J. D. Oña, “Cluster Analysis for diminishing heterogeneous opinions of service quality public transport passengers," Procedia-Social and Behavioral Sciences, vol. 162, pp. 459-466, 2014.

[11] C. F. Fang, L. Elefteriadou, K. K. Pecheux, and M. T. Pietrucha, "Using fuzzy clustering of user perception to define levels of service at signalized intersections," Journal of Transportation Engineering, vol. 129, no. 6, pp. 657-663, 2003.

[12] C. F. Fang and K. K. Pecheux, "Fuzzy data mining approach for quantifying signalized intersection level of services based on user perceptions," Journal of Transportation Engineering, vol. 135, no. 6, pp. 349-358, 2009.

[13] L. Dell'Olio, A. Ibeas, and P. Cecín, "Modelling user perception of bus transit quality," Transport Policy, vol. 17, no. 6, pp. 388-397, 2010.
[14] J. D. Oña, R. D. Oña, L. Eboli, and G. Mazzulla, "Heterogeneity in perceptions of service quality among groups of railway passengers," International Journal of Sustainable Transportation, vol. 9, no. 8, pp. 612-626, 2015.

[15] Y.-H. Cheng and K.-C. Liu, "Evaluating bicycle-transit users' perceptions of intermodal inconvenience," Transportation Research Part A: Policy and Practice, vol. 46, no. 10, pp. 1690-1706, 2012.

[16] Y.-H. Cheng and S.-Y. Chen, "Perceived accessibility, mobility, and connectivity of public transportation systems," Transportation Research Part A: Policy and Practice, vol. 77, pp. 386-403, 2015.

[17] M. Bordagaray, L. Dell'Olio, A. Ibeas, and P. Cecín, “Modelling user perception of bus transit quality considering user and service heterogeneity," Transportmetrica A: Transport Science, vol. 10, no. 8, pp. 705-721, 2014.

[18] N. Lathia, C. Smith, J. Froehlich, and L. Capra, "Individuals among commuters: building personalised transport information services from fare collection systems," Pervasive and Mobile Computing, vol. 9, no. 5, pp. 643-664, 2013.

[19] K. Qiao, P. Zhao, and J.-X. Wen, "Passenger market segmentation of high-speed railway based on latent class model," Journal of Transportation Systems Engineering and Information Technology, vol. 17, no. 2, pp. 28-34, 2017.

[20] M. G. Bellizzi, L. Dell'Olio, L. Eboli, and G. Mazzulla, "Heterogeneity in desired bus service quality from users' and potential users' perspective," Transportation Research Part A: Policy and Practice, vol. 132, pp. 365-377, 2020.

[21] D. A. Linzer and J. B. Lewis, "poLCA: an R package for polytomous variable latent class analysis," Journal of Statistical Software, vol. 42, no. 10, pp. 1-29, 2011.

[22] S. Askari, N. Montazerin, and M. H. F. Zarandi, "Generalized possibilistic fuzzy C-means with novel cluster validity indices for clustering noisy data," Applied Soft Computing, vol. 53, pp. 262-283, 2017.

[23] S. Das and D. Pandit, "Determination of level-of-service scale values for quantitative bus transit service attributes based on user perception," Transportmetrica A: Transport Science, vol. 11, no. 1, pp. 1-21, 2015. 